\title{
I NFORMES
}

\section{LA ENSEÑANZA DE LA DEMOGRAFIA EN AMÉRICA LATINA *}

\section{ANTECEDENTES}

1. Hace poco más de diez años se afirmó: "La mayoría de las universidades en América Latina no incluyen cursos de demografía en sus curricula".. Esta afirmación ha perdido actualidad de entonces a la fecha debido al desarrollo de actividades en investigación y enseñanza demográficas. No obstante, si se hace referencia a la enseñanza especializada, el avance ha sido mínimo.

2. Si sólo se hace referencia a la enseñanza de la demografía en los programas de estudio de diversas especialidades, son pocas las universidades latinoamericanas en donde no se enseña demografía. Sin embargo, la manera en que se enseña no es siempre la más adecuada y no se transmiten a los estudiantes los avances logrados en los últimos años, respecto al mayor conocimiento de las diversas situaciones demográficas y al desarrollo de metodologías y técnicas de medición y análisis.

3. En los últimos diez años, tanto en América Latina como en las demás regiones, además de la expansión relativamente importante de la enseñanza e investigación demográficas y de los recursos necesarios para un mayor avance, ha habido paralelamente una expansión de actividades orientadas a discutir y difundir las condiciones y los posibles efectos de la dinámica de la población. A este respecto se ha planteado la necesidad de políticas en donde las variables demográficas estén ciebidamente consideradas, y se ha subrayado que "una política de población no debe adoptarse en forma aislada o unilateral sino como parte del desarrollo total". 2

4. Sin embargo, no es éste el espíritu de todas las reuniones que se realizan cada vez en mayor número; en otras, los planteamientos parten más de la necesidad inmediata de control de la natalidad, que se afirma, facilitará un mayor desarrollo económico en América Latina. ${ }^{3}$

5. Si se considera el conjunto de actividades en torno a la demografia (creación de centros de enseñanza e investigación, avances importantes en la elaboración de información básica, reuniones nacionales e internacionales sobre los problemas de población desde diversos puntos de vista o en relación con diversas disciplinas, etc.); si se tiene en cuenta el interés y la inquietud con que los gobiernos y las instituciones consideran los problemas relacionados con la dinámica de la población y a su vez los avan-

* Trabajo preparado por el Centro de Estudios Económicos y Demográficos del Colegio de México para la primera reunión del Comité Asesor de Población de la OEA, Washington, julio 29-31 de 1968. En este trabajo se pretende dar una visión general de la enseñanza de la demografía en América Latina. Un trabajo completo requeriria de mayor información.

$1 \mathrm{E}$. Dieulefait, "The Teaching of Demography in Latin America", en UNESCO, The University Teaching of Social Sciences: Demography, Paris, 1957.

2 Reunión sobre Políticas de Población en Relación al Desarrollo en América Latina (Caracas, 11-16 de septiembre de 1967), Informe final, Washington, Unión Panamericana, 1967, p. 7. (Doc. UP/Ser.H/V/REPO/II/17 Rev.)

3 VIII Conferencia de la Federación Internacional de Planificación de la Familia, Santiago, 1967. 
ces de la demografía como disciplina independiente y en relación con otras áreas de estudio, los últimos diez años han sido decisivos en la expansión de la demografía y los estudios de población en todo el mundo.

En América Latina estas actividades han siclo fructíferas pero son todavía insuficientes.

\section{LA ENSEÑANZA DE LA DEMOGRAFİA}

6. En los ciclos primario y secundario de enseñanza, los conocimientos demográficos que se imparten son muy limitados y en general se reducen a menciones del volumen y distribución de la población en los países, en las regiones y en el mundo. En algunos países en donde se han agregado cursos sobre problemas económicos, políticos y sociales a nivel secundario (México, Chile) se incluyen descripciones de los cambios demográficos y consideraciones generales sobre su relación con algunos aspectos del desarrollo. En casi todos los países, dentro de los cursos de geografía humana, se da especial atención a los aspectos demográficos. En otros, como introducción a la sociología, a la antropología e incluso en cursos de "civismo", generalmente se hace mención de algunos aspectos demográficos.

7. A nivel universitario, la enseñanza formal de la demografía no existía en ningún país de Latinoamérica. Sólo se impartían algunos cursos generales incorporados a los programas de enseñanza de ciencias actuariales, economía y salud pública, como es el caso de algunas universidades de Argentina, Brasil y México. En la actualidad, en la mayor parte de las universidades en las carreras de economía, sociología, antropología y actuaría, la demografía se ha incorporado como asignatura en los programas de estudio. En estos casos los cursos tienen carácter general, están orientados a problemas de medición de indicadores demográficos sencillos, al estudio de las tendencias demográficas, al estudio general de doctrinas y teorías de población y a la consideración de problemas demográficos relacionados con la especialidad en que se imparte. A este nivel, los problemas demográficos han cobrado mayor importancia cuando se ponen en relación con los económicos, sociales, educativos, de salud, etc. ${ }^{4}$

8. Sin embargo, y no obstante una mayor participación de profesores que han recibido adiestramiento especializado en demografía, la enseñanza de esta disciplina en las carreras mencionadas aún no se imparte al nivel adecuado por múltiples razones: calidad deficiente de la enseñanza en general en las escuelas y facultades universitarias, insuficiencia de profesores con adecuada formación, falta de interés por parte de los alumnos e incluso de los profesores, falta de reconocimiento de la demografía como disciplina independiente, desconocimiento de los avances en demografía y en estudios de población, confusión de la demografía con política de población o con planeación familiar, etc.

9. Junto con la expansión de la enseñanza en las diversas carreras y particularmente en ciencias sociales, se están creando departamentos en algunas universidades, ya sea en relación con problemas económicos, sociales, de salud o de programación, orientados a la investigación y a la enseñanza de la demografía (Colombia, Venezuela, Brasil, México, Centroamérica).

10. La enseñanza de la demografía fue fundamentalmente una tarea realizada a través de "cursos de adiestramiento internacionales, general mente de corta duración. El IASI, por ejemplo, organizó entre los afios 1954 y 1960 cuatro centros subregionales para la América Central y los países

4 Naciones Unidas, Consejo Económico y Social, Reglional Demographic Programmes under Changing Conditions, Interregional Workshop, Elsinore, Denmark, $19-30$ de junio de 1967 . (Doc. E/CN.9/CONF.4/L.2.). 
del Caribe dedicados al adiestramiento en estadística. El Centro Interamericano de Enseñanza de Estadística Económica y Financiera (CIEF), creado en 1953, incluyó un curso sobre Elementos de Estadísticas Demográficas. Aunque valiosos, estos cursos no eran precisamente el mejor medio para solucionar el problema de personal especializado en demografía".s

11. Una mención especial debe hacerse de la enseñanza de la demografía en las escuelas de salud, en donde generalmente se incluían cursos de demografía más bien relacionados con aquellos especializados en bioestadística. En la actulalidad se da generalmente en estas escuelas mayor importancia a la demografía y en algunos casos, por ejemplo en Chile, descle hace tiempo se dan cursos de demografía y se realiza investigación.

12. A partir de 1953 se inician actividades orientadas a la formación de personal especializado, con la creación en marzo de 1953 del Centro Interamericano de Bioestadística, que impartió cursos de demografía y estadísticas vitales, dando mayor énfasis a la recolección, elaboración y publicación de información y a algunos aspectos de análisis demográfico. ${ }^{6}$ Desgraciadamente, la duración de este Centro sólo fue de tres años.

13. Un segundo paso en la enseñanza especializada a nivel regional lo constituye sin duda la formación del Centro Latinoamericano de Demografía (CELADE), uno de los centros regionales de las Naciones Uniclas que funciona en la Universidad de Chile a partir de 1957.

La labor de enseñanza de este Centro constituye la experiencia más importante y fructífera tanto en materia de enseñanza como en investigación. Su influencia ha sido considerable y sostenida y como resultado de sus actividades se ha incrementado notablemente la difusión del conocimiento de los problemas demográficos. Su labor se ha reflejado en otros centros internacionales lo mismo que en actividades nacionales de muy diversa naturaleza relacionadas de una forma u otra con el estudio sistemático de la demografía y los problemas de población.

Las actividades de enseñanza y capacitación se realizan a través de un programa que comprende un curso básico, un curso avanzado y otro de especialización.

El curso básico, con duración de diez meses y medio, está destinado a cubrir diversos temas de la demografía a través de clases teóricas y prácticas y de seminarios. Se dedica parte de los dos últimos meses a la presentación de un trabajo final de investigación de alcance limitado.

En un segundo año, destinado solamente a los estudiantes más aventajados, con interés y vocación, se profundizan los conocimientos en el campo de la demografía siguiendo cursos académicos y participando en las actividades de investigación. Se agregan en este año, además de currsos más especializados en demografía, nociones de muestreo, análisis sobre los problemas de crecimiento demográfico y cambio social y sobre recursos humanos y programación del desarrollo, así como métodos y técnicas de investigación social. En este año se amplía particularmente el análisis de la mortalidad y la fecundidad.

En un tercer año, los estudiantes sobresalientes continúan participando en las investigaciones que realiza CELADE y a la vez colaboran en las tareas de docencia. Este tercer año tiene por objeto la formación de persint

5 Carmen A. Miró, Experiencias y problemas en la promoción de la enseñanza y la investigación demográficas en los paises en vias de desarrollo: el caso de América Latina, trabajo presentado en la Scgunda Conferencia Mundial de Población, Belgrado, agosto de 1965. El CIEF funcionó bajo patrocinio de la Organización de los Estados Americanos.

"Ibill. y "Centro Interamericano de Bioestadística: Programa para 1954", Prospecto N: 2, Santiago. 
sonal para la enseñanza especializada de la demografía y para la prestación de asistencia técnica nacional e intermacional.

A fines de 1967, habían recibido adiestramiento 160 becarios de los distintos países latinoamericanos y en 1968 lo están recibiendo 23, distribuidos en la siguiente forma:

\begin{tabular}{lcc}
$\begin{array}{l}\text { Permanencia } \\
\text { hasta de }\end{array}$ & $\begin{array}{c}\text { Becarios } \\
1958 \text { a } 1967\end{array}$ & $\begin{array}{c}\text { Becarios } \\
\text { en } 1968\end{array}$ \\
Un año & 110 & 16 \\
Dos años & 39 & 6 \\
Tres años & 11 & 1 \\
\multicolumn{1}{c}{ Total } & 160 & $\frac{1}{23}$
\end{tabular}

La distribución de los becarios por países, considerando los .160 hasta 1960 y los 16 clel primer año de 1968, o sea 176, es la siguiente:

$\begin{array}{lrlr}\text { Argentina } & 23 & \text { Haití } & 3 \\ \text { Brasil } & 10 & \text { Honduras } & 5 \\ \text { Bolivia } & 4 & \text { México } & 13 \\ \text { Colombia } & 18 & \text { Nicaragua } & 1 \\ \text { Costa Rica } & 6 & \text { Panamá } & 1 \\ \text { Cuba } & 1 & \text { Paraguay } & 5 \\ \text { Chile } & 25 & \text { Perú } & 13 \\ \text { Rep. Dominicana } & 5 & \text { Puerto Rico } & 3 \\ \text { Ecuador } & 10 & \text { Uruguay } & 5 \\ \text { El Salvador } & 8 & \text { Venezuela } & 7 \\ \text { Guatemala } & 4 & & \end{array}$

Además, la subsede de CELADE ha iniciado cursillos en Costa Rica, de preparación previa para los estudiantes centroamericanos que posteriormente realizarán estudios en Santiago.

14. El impacto que los egresados del CELADE han tenido en el desarrollo de la enseñanza e investigación demográficas en sus países ha aumentado considerablemente en los últimos años. Además de su participación en los estudios demográficos propiamente dichos, se espera de ellos una intensificación de su actividad enfocada hacia los problemas cle población y salud pública, los problemas de educación y formación de mano de obra y los programas de desarrollo económico y social. Puede afirmàrse que la mayor parte de los trabajos de investigación recientes, provienen de los investigadores, becarios y egresados del CELADE, o bien de organismos regionales como es el caso de la CEPAL.

15. Los trabajos que han sido dirigidos por el CELADE y que han despertado el interés por estos problemas en la región, se han orientado hacia la medición de los índices demográficos básicos de cada país, así como a proporcionar estimaciones de población futura que sirvan a los gobiernos y las oficinas de planificación en sus programas de desarrollo. En la elaboración de estos trabajos han sido abordados temas como el análisis critico de la información básica; estudios sobre la mortalidad por causas y tablas de vida; estimaciones de las tasas de fecundidad por edad e índices de reproducción y tablas de nupcialidad; migraciones interiores y urbanización; tasas de participación en la actividad económica y tablas cle vida activa. Muchos cle los trabajos incorporan aportaciones valiosas de carácter metodológico.

Asimismo, y como actividad lateral que en cierta forma ha permitido 
el mayor adiestramiento de egresados o de investigadores en ciencias sociales, además del valor que representan por la nueva aportación de información, el CELADE ha llevado a cabo diversas encuestas demográficas en forma conjunta con instituciones nacionales. Se destacan las encuestas comparativas de fecundidad urbana, que se llevaron a cabo en siete ciudades latinoamericanas y a las cuales se agregaron posteriormente tres ciudades más; las encuestas que ahora se realizan en cinco países sobre fecundidad rural; las encuestas de migración en tres ciudades; la encuesta demográfica experimental de Guanabara en Brasil, destinada a obtener periódicamente información fidedigna y detallada sobre fecundidad, mortalidad, situación en materia de empleo, desempleo y subempleo, estructura del consumo familiar, movilidad social y profesional y la asistencia escolar; la encuesta demográfica experimental de Cauquenes en Chile, que busca la recolección de datos que permitirán la elaboración de los índices demográficos más elementales a costos razonables; y las encuestas sobre aborto inducido que se realizan en varios países.

16. La enseñanza especializada en centros nacionales es muy reciente. Hasta ahora existe en México, y hay proyectos de establecerla en Brasil, Colombia y Venezuela.

En 1964 comenzó a funcionar el Centro de Estudios Económicos y Demográficos de El Colegio de México. Constituye el primer centro nacional de enseñanza e investigación especializado en demografía en la región. En este centro se otorgan las maestrías en economía, demografía y estadística después de dos años de estudios. En demografía los cursos tienen una doble orientación: técnicas y análisis demográfico y teoría y análisis económico, incluyendo cursos de desarrollo económico y de sociología del desarrollo. Con la finalidad de actualizar los conocimientos de aquellos estudiantes que desean ingresar a los cursos, especialmente en matemáticas e idiomas, se imparte un curso preparatorio de un semestre.

Las promociones son cada dos años. A la fecha en la generación 1964 1965, de 8 estudiantes que iniciaron, 5 terminaron sus estudios; en la generación 1966-1967 fueron admitidos 8 estudiantes y terminaron 4; en la generación 1967-1968-1969 (se adelantaron los cursos para posibilitar el cambio de calendarios), ingresaron doce alumnos de los cuales continúan a la fecha 8. En esta última generación realizan estudios dos estudiantes del Perú.

17. En demografía, el Centro dispone de cuatro profesores e investigadores de tiempo completo y dos de medio tiempo. El CELADE, el Instituto Nacionales de Estudios Demográficos de Francia (INED) y otros centros de universidades norteamericanas han colaborado con el cuerpo de profesores en las actividades de enseñanza.

18. Los cursos a crearse en otras partes serán en el Instituto Brasileiro de Geografía y Estadística, en la Facultad de Economía de la Universidad Central de Venezuela y en el Centro de Estudios sobre Desarrollo Económico de la Universidad de los Andes de Colombia.

19. La investigación demográfica en centros nacionales también es de fecha reciente. En el caso de El Colegio de México, está orientada al estudio de los efectos del crecimiento de la población sobre el desarrollo. Se trabaja en temas tales como migración interna, proceso de urbanización, cambios en la estructura sectorial de la mano de obra y problemas relativos a la previsión de necesidades tanto de educación como de mano de obra. Por otra parte, conjuntamente con el Instituto de Investigaciones Sociales de la Universidad Nacional Autónoma de México, se realiza una encuesta de fecundidad rural, y se programa la realización de un estudio sobre migra. ción y estructura ocupacional, con la cooperación técnica de las Naciones Unidas. 
20. En otros países se llevan a cabo investigaciones en institutos y centros diversos como es el caso del Centro de Investigaciones Sociales del Instituto Torcuato Di Tella en Argentina, del Instituto de Estudios Peruanos, del Centro de Estudios de Población y Desarrollo en Perú, del Centro de Estudios del Desarrollo de la Universidad Central de Venezuela y otros.

21. Debido a la insuficiente investigación demográfica en las universidades latinoamericanas, las instituciones gubernamentales han establecido unidades de análisis demográfico, principalmente en las oficinas nacionales de estadística o bien en las de planificación. Países como Argentina, Brasil, Colombia, Costa Rica, Chile, Ecuador, Honduras, Panamá, Perú y Venezuela han formado recientemente unidades de estudios demográficos y elaboran trabajos útiles para el conocimiento de problemas sociales, económicos, educacionales, de vivienda, de servicios públicos, etc., en donde la población ejerce su influencia. También se efectúan análisis críticos de la información básica demográfica, tablas de vida, proyecciones de población, etc. El CELADE asesora permanentemente a estos grupos.

22. La investigación demográfica también ha sido promovida por los médicos. Las escuelas de medicina y de salubridad de la Universidad de Chile han venido trabajando en estudios de mortalidad y planificación de la familia. En este país se ha desarrollado una actividad muy encomiable en materia de enseñanza a médicos e investigación de problemas de población y salud. La Asociación Colombiana de Facultades de Medicina estableció una División de Estudios de Población en donde se han hecho estudios sobre problemas demográficos, entre ellos sobre aborto induciclo. Dicha Asociación participa actualmente en la encuesta de fecundidad rural en colaboración con el CELADE. En Colombia particularmente, los médicos han sido muy activos no sólo en materia de investigación, sino también, últimamente, publicando traducciones de trabajos relativos a análisis demográfico, fecundidad y planeación familiar.

\section{ACTIVIDADES OUE CONTRIBUYEN AL DESARROLLO DE LA ENSEÑANZA Y LA} INVESTIGACIÓN DEMOGRAFICAS EN AMÉRICA LATINA

23. Sin pretender que las menciones anteriores sean completas - constituyen más bien una revisión del material disponible y el conocimiento de lo que realizan algunas instituciones-, es pertinente mencionar la manera cómo en la actualidad, dentro y fuera de América Latina, ellas promueven directa o indirectamente actividades que en breve permitirán un mayor desarrollo de la demografía y los estudios de población.

24. Del 23 de marzo al 5 de abril de 1965, se realizó la decimotercera reunión de la Comisión de Población de las Naciones Unidas.7 El Consejo Económico y Social, tomando en cuenta las resoluciones de la Asamblea General sobre crecimiento de la población y desarrollo económico (18 de diciembre de 1962), y las conclusiones del Consejo (5 de abril de 1963) sobre intensificación de estudios, los problemas de desarrollo asociados con el crecimiento y la estructura de la población, las respuestas de los gobiernos sobre los problemas resultantes de la interacción entre desarrollo económico y cambios demográficos, la carencia de personal capacitado en los distintos países y la necesidad de intensificar y extender los trabajos de las Naciones Unidas y los organismos especializados, apoyó las recomendaciones de la Comisión en cuanto al incremento y mejora de estadísticas demográficas, el fortalecimiento de las actividades de los centros regionale!

7 Naciones Unidas, Comisión de Población, Informe de la decimotercera sesión Nueva York, 1965. (Doc. E/4019 E/CN.9/202.) 
de enseñanza e investigación demográfica y otras actividades que tienden a formar personal técnico calificado en los países en desarrollo, expansión e intensificación de la investigación y trabajo técnico, aumento de la asistencia técnica a los gobiernos que lo soliciten y realización de conferencias y actividades afines en materia de población.

25. Del 30 de agosto al 10 de septiembre de 1965 se llevó a cabo en Belgrado la Segunda Conferencia Mundial de Población. En esta reunión se puso énfasis en los estudios sobre la interrelación entre las características de la estructura económica y el crecimiento de la población en países en desarrollo. La participación de expertos de los países en desarrollo fue considerable, entre ellos alrededor de 80 latinoamericanos.

26. Desde agosto de 1957, cuando fue fundado, hasta abril de 1966, el CELADE funcionó como proyecto de Ia Oficina de Asistencia Técnica de las Naciones Unidas, teniendo además apoyo financiero del Consejo de Población y en el último período de la Fundación Ford. Desde mayo de 1966 hasta abril de 1971, el Centro está siendo financiado por el Fondo Especial de Asistencia para el Desarrollo de las Naciones Unidas y por los aportes de los gobiernos latinoamericanos que suscriben el Plan de Operaciones destinado a ampliar e intensificar sus actividades. Hasta junio de 1968, este plan ha sido firmado por trece gobiernos de la región. 8

La labor desarrollada por el CELADE en muchos sentidos se ha convertido en un ejemplo de funcionalidad y adecuación a las necesidades regionales. Su influencia ha estimulado el desarrollo de la actividad científica en materia demográfica.

27. Del 19 al 30 de junio de 1967 se realizó en Elsinore, Dinamarca, una reunión del Grupo de Trabajo Interregional sobre Programas de Formación en Materia Demográfica, organizada por la Comisión de Cooperación Técnica y por la División de Población del Departamento de Asuntos Económicos y Sociales de las Naciones Unidas en colaboración con el Gobierno de Dinamarca. 9

En las discusiones se partió del hecho de que la demografía y el estudio de la población son de carácter multidisciplinario, ya que las tendencias demográficas dependen de diversas condiciones y del desarrollo de fenómenos múltiples; que la demografía se ha establecido recientemente como disciplina separada en las universidades; que esta disciplina ha sido especialmente dinámica durante los años recientes debido, entre otras cosas, a la adopción de políticas de población en muchos países; y que tanto en el ámbito nacional como internacional, hay muchas y diversas condiciones institucionales que en muchos casos obstaculizan y en otros estimulan al avance de la enseñanza y de la investigación en materia de población. Se señaló que varios razones determinan la necesidad creciente de ampliar la capacidad en materia de demografía. Además de la necesidad de expertos para levantamiento de censos, organización de estadísticas, análisis demográfico básico, sanidad pública, educación, etc., han surgido nuevas exigencias. La formulación de políticas económicas y sociales y la planificación del desarrollo nacional requieren análisis demográficos y estudios de población más extensos y profundos.

Entre otras muchas conclusiones a que llegó el grupo, se consideró que si la demografía se habría de establecer por derecho propio en las universidades, debía tratársela como disciplina y atribuírsele la condición de tal, teniendo debidamente en cuenta las consecuencias que esto acarrearía. Se

8 CELADE, Origen, organización y objetivos, Santiago, 1968.

9 Naciones Unidas, Informe del Grupo de Trabajo Interregional sobre programas de formación en materia demográfica, Elsinore, Dinamarca, 19 a 30 de junio de 1967, Nueva York, 1967. (Doc. ST/TAO/SER.c/98/E/CN.9/207-E/CN.9/CONF. 4/1.) 
señaló la falta de coordinación entre diversos programas de formación. Se indicó que era necesario organizar la formación en materia demográfica de conformidad con los requisitos básicos de cada país. Se estimó la importancia que en diversos paises ha tenido la formación de especialistas en centros independientes $\mathrm{y}$, al mismo tiempo, se destacó que estos centros debían de tratar de establecer relaciones con los departamentos correspondientes de las universiclades nacionales. Se expresó la esperanza de que se establecieran departamentos de demografía.

A su vez se determinaron ciertas normas y niveles considerados necesarios para la formación de demógrafos, como especialización o como complemento de la formación en materias afines. Otros asuntos planteados fueron la mejor utilización de los demógrafos y de los expertos en población en el plano nacional, los problemas de los centros regionales, la formación en materia de planificación de la familia. Se hizo hincapié en la necesidad de coordinación entre los programas nacionales y regionales en materia demográfica.

28. La UNESCO convocó a un Comité especial de expertos, los que se reunieron en París del 6 al 12 de julio de 1967 para analizar las responsabilidades de este organismo en relación con los problemas de población y la posibilidad de realizar un intercambio fructífero de información en materia demográfica. De las discusiones se llegó a la conclusión de que los problemas demográficos no necesariamente requieren soluciones demográficas, ya que se plantean dentro de un marco complejo y las soluciones son también complejas y demandan cambios económicos y sociales.

El grupo adoptó, entre otras, las siguientes recomendaciones: $a$ ) intensificar los estudios que expliquen las interrelaciones entre educación y cultura por una parte, y las tendencias de la población por otra; $b$ ) introducir en los programas regulares de enseñanza elementos de demografía (señalando varios caminos para lograrlo); y c) otorgar becas para la formación de profesores de demografía.

29. Bajo los auspicios de la Organización de los Estados Americanos, la Organización Panamericana de la Salud, el Consejo de Población, el Instituto Aspen para Estudios Humanísticos y con la colaboración del Gobierno de Venezuela, se llevó a cabo en Caracas del 11 al 16 de septiembre de 1967 la Reunión sobre Políticas de Población en Relación al Desarrollo en América Latina.

Respecto a la investigación y enseñanza de la demografía se recomendó el estímulo para la formación de personal, en especial la capacitación de demógrafos a los diversos niveles necesarios; la remuneración adecuada de estos técnicos; la institucionalización permanente de 'la investigación y la enseñanza que asegure su continuidad y fortalecimiento; realización de esfuerzos orientados a la investigación de las variables que determinan los cambios demográficos; el perfeccionamiento de las metodologías y técnicas de investigación, con la participación de expertos, principalmente demógrafos, economistas y sociólogos; la mayor cooperación financiera, de becas y de asistencia técnica; el estímulo del intercambio eficaz y la cooperación científica en el campo de la investigación demográfica con los países desarrollados.

30. En la Reunión del Comité Directivo del Consejo Latinoamericano de Ciencias Sociales celebrada en Buenos Aires del 3 al 5 de junio de 1968, se acordó la creación de una comisión de trabajo para estimular y coordinar la investigación y los estudios demográficos de los diversos centros latinoamericanos interesados con la cooperación del CELADE. En forma resumida, los objetivos de esta comisión son: a) contribuir a que se logre en los paises un mayor desarrollo y aprovechamiento de las fuentes de información; b) promover un mayor intercambio de información en materia de investigación demográfica; c) estimular el intercambio más adecuado 
de los avances metodológicos y asegurar así el progreso de la investigación y la enseñanza de la demografía; a) colaborar en la creación de centros nacionales de enseñanza e investigación demográficas; e) promover reuniones de cooperación y discusión, orientadas a la realización de proyectos concretos de investigación; f) cuidar que a la vez que se logren avances en la enseñanza y en la investigación en demografía, su desarrollo esté cada vez más interrelacionado con otras ciencias sociales; $g$ ) favorecer la elaboración de marcos teóricos aplicables a la realidad de la región.

31. Del 23 al 26 de junio de 1968 se celebró en Bogotá la Conferencia Panamericana sobre la Enseñanza de la Demografía en las Facultades de Medicina. Una conferencia de esta naturaleza no tenía precedente en la historia de la educación de la medicina en la región. ${ }^{10}$

Esta conferencia fue a su vez el resultado de reuniones anteriores en donde a niveles nacionales, se discutió en las asociaciones de escuelas y facultades de medicina sobre las siguientes interrogantes: ¿Se justifica la enseñanza de la demografía en las escuelas y facultades de medicina? ¿Qué debe enseñarse de demografía? ¿Con qué profundidad debe enseñarse cada tema? ¿Cuál es la mejor ubicación de la enseñanza de la demografía dentro de los programas de enseñanza de medicina? ¿Cómo debe enseñarse, con qué material docente y quién debe impartir la enseñanza? ¿Qué investigaciones en la problemática de salud y demografía deberían realizarse?

A esta Conferencia Panamericana asistieron representantes de todas las asociaciones de escuelas y facultades de medicina y representantes de las cuatro entidades copatrocinadores de la reunión (la Federación Panamericana de Asociaciones de Facultades de Medicina, el Centro Latinoamericano de Demografía, la Organización Panamericana de la Salud y la Asociación Colombiana de Facultades de Medicina), aparte de invitados de fundaciones, organizaciones mundiales, centros nacionales, etc.

El informe final consigna que para el ejercicio de la medicina integral, es necesario el conocimiento de la población sobre la cual se trabaja, de aquí que el estudiante de medicina deba conocer las fuentes básicas de los datos y sus limitaciones, ya que él mismo es un recolector y consumidor de datos. Se analizan en el informe aquellas variables demográficas que deben considerarse en la enseñanza a médicos y los temas específicos que deberían tratarse, la profundidad adecuada y su ubicación dentro de los programas de medicina.

El CELADE presentó a la reunión un documento en donde se indican detalladamente los cursos necesarios, su justificación, su desglose por temas específicos y se plantea la necesidad de investigación con-carácter multidisciplinario. 11

32. Otras actividades que están relacionadas con los problemas de enseñanza e investigación en demografía son, por ejemplo, el Seminario sobre la Situación Demográfica en Centroamérica, en julio de 1967, patrocinado por la ODECA y el CELADE; el Seminario sobre Organización y Levantamiento de Censos de Población y Habitación para América Latina celebrado del 20 al 31 de mayo de 1968 en Santiago con relación a los censos de 1970 , al cual asistieron los directores o representantes de las direcciones de estadística de los diversos países y que fue organizado por la Comisión Económica para la América Latina y la Ofícina de Cooperación Técnica de las Naciones Unidas; el Seminario de Población y Trabajo en Honduras realizado del 13 al 17 de mayo de 1968.

10 Para mayor detalle, ver: Conferencia Panamericana sobre la Enseñanza de la Demografía en las Facultades de Medicina (Bogotá junio 23-26 de 1968) Relato final, y CELADE, Informativo de CELADE, Vol. VIII, Núm. 3.

11 Jorge García, Enseñanza de la demografía en las escuelas (facultades) de medicina, Santiago, CELADE, 1968. (Serie A, Núm. 87.) 
IV. Problemas, NECesidades Y OBJETIVOS QUE DEBEN CUBRIRSE A TRAVÉS DE L.A ENSEÑANZA E INVESTIGACIÓN DEMOGRÁFICAS

De esta breve revisión se desprenden algunas conclusiones generales:

Si bien en América Latina en los últimos años se ha intensificado la enseñanza y la investigación demográficas, aún es insuficiente y solamente se ha cubierto una fase inicial caracterizada por la existencia de un número de técnicos preparado en el CELADE (los más) o en otras universidades, principalmente norteamericanas. Puede afirmarse que en gran parte de las universidades latinoamericanas ya se enseña demografía, como materia dentro de los programas de diversas especialidades, particularmente en ciencias sociales y actuaría. A la fecha sólo existe en la región el CELADE como centro regional de enseñanza e investigación especializada, y un centro nacional, el Centro de Estudios Económicos y Demográficos de El Colegio de México; en los últimos dos años se han organizado centros en universidades de Brasil y de Colombia que darán instrucción en demografía y que por ahora realizan actividades de investigación; otro se está organizando actualmente en Venezuela. Existe conciencia entre los gobiernos de la necesidad de conocimiento de los problemas de población en relación con el desarrollo y la educación, la salud, la vivienda, etc. Se realizan múltiples actividades por organismos internacionales y regionales, universidades, asociaciones, centros diversos, etc., que dentro y fuera de la región promueven activamente la enseñanza y la investigación demográficas, principalmente en las universidades y tendiendo a la existencia, como disciplina independiente, de la carrera de demógrafo. Existen grupos profesionales muy activos, particularmente los médicos que han desarrollado una labor sobresaliente para lograr que la demografía sea incluida dentro de los programas de enseñanza.

34. Los diversos grupos en los que se ha discutido la necesidad de satisfacer los requerimientos en materia de enseñanza e investigación de la demografía y los problemas de población, han coincidido en la mayor parte de sus conclusiones y recomendaciones. Conviene mencionar algunas de las más sobresalientes, de manera resumida, las cuales, por otra parte, han sido expuestas y están contenidas en los diversos documentos mencionados a lo largo de este trabajo.

\section{Difusión y enseñanza de la demografia y problemas de población}

a) Se ha indicado la necesidad de difundir por diversos medios los conocimientos demográficos relacionados con aspectos de la problemática social, en la enseñanza primaria, secundaria, pre-universitaria, en la enseñanza de adultos y en las universidades (UNESCO).

b) Se ha señalado como primera necesidad la de instruir a aquellos que llegarán a ser los profesores de demografía en los diversos niveles y dentro de las diversas carreras profesionales (CELADE).

c) La formación de profesionales capaces de llevar a cabo investigación independiente, requiere de niveles elevados de especialización en la enseñanza de la demografía, tanto como disciplina independiente como en relación con otras disciplinas, dado el carácter multidisciplinario de los problemas de población (grupos de trabajo de las Naciones Unidas, UNESCO, CELADE, El Colegio de México, OEA y otros).

d) Si bien por muy diversas circunstancias y posibilidades nacionales, la enseñanza especializada de la demografía se imparte con frecuencia en centros independientes, debe tenderse a que se lleve a cabo también en el seno de las universidades (Naciones Unidas, CELADE, El Colegio de México), 
e) Dentro de los diversos niveles deseables en la formación de un demógrafo se han señalado un primer nivel dedicado a la enseñanza de la demografía descriptiva (Naciones Unidas, Elsinore), o análisis demográfico básico (CELADE); un segundo nivel en donde se estudiarían los métodos $\mathrm{e}$ instrumentos analíticos necesarios para calcular los índices demográficos, los cambios de las tendencias y estructuras demográficas, etc. (Naciones Unidas, Elsinore), que comprende parte del análisis demográfico avanzado que se imparte en el CELADE; un tercer nivel estaría dedicado al estudio de diversos temas especializados como planificación económica y social, educación, salud, etc., el cual en el CELADE corresponde a parte del segundo y tercer años de enseñanza.

f) El CELADE a su vez consiclera la necesidad de cursos especializados en métodos de recolección y elaboración de datos demográficos, incluyendo el muestreo, para el personal que se dedique a esas tareas. Esta propuesta tiene como antecedentes los cursillos que se han realizado. con motivo de los censos de 1950 y 1960 , salvo que ahora se propone un mayor grado de especialización y duración.

g) En general se ha recomendado la enseñanza de la demografía en relación muy estrecha con diversas disciplinas sociales, particularmente con la economía y la sociología. En El Colegio de México la enseñanza de la demografía se imparte en íntima relación con la economía y en las universidades norteamericanas tradicionalmente ha estado ligada a la sociologia. En Europa la relación más frecuente ha sido con la economía. Este tipo de orientación de la enseñanza y de la investigación, particularmente en los países en desarrollo, se considera adecuada a las necesidades.

h) En todas las reuniones se ha señalado la necesidad de disponer de más becas, de una mayor contribución financiera por parte de instituciones públicas y privadas a los centros de enseñanza e investigación, mayor colaboración internacional, etc. Los recursos nacionales en muchos casos resultan insuficientes para resolver los problemas que presenta la formación de personal calificaaio.

i) Se ha indicado también la dificultad de atraer estudiantes y graduados universitarios, entre otras razones, por los métodos aún ineficaces para el reclutamiento de candidatos, la falta de personal especializado, e1 desconocimiento de la demografía como disciplina independiente, la falta de material bibliográfico en español y la falta de demanda y reconocimiento del demógrafo como profesional (CELADE).

\section{Problemas y necesidades en la investigación demográfica}

a) Se ha llamado la atención acerca de que en muchos de los paises en desarrollo hay escasez de personal capacitado en demografía, observándose al mismo tiempo una utilización insuficiente e inadecuada de los expertos (Naciones Unidas, Elsinore). Este problema, que afecta también a otras disciplinas, implica generalmente la utilización del experto como administrador, carencia de recursos para realizar investigaciones, falta de personal auxiliar, así como incomprensión, por parte de directores o funcionarios.

b) El CELADE, en un documento presentado a la Segunda Conferencia Mundial de Población, refiriéndose a la falta de tradición latinoamericana con respecto a la investigación, dijo: "...se señaló que la investigación social está sólo comenzando en las universidades de la región. El éxito en la promoción de la investigacíón demográfica, confinada hoy día en gran medida a las instituciones gubernamentales, dependerá inter alia de que a la demografía se le otorgue el reconocimiento anteriormente discutido, 
de la disponibilidad de personal capaz de planear y ejecutar determinados proyectos de investigación y del apoyo financiero que se le conceda".12

c) El Colegio de México, al presentar al Comité Directivo del Consejo Latinoamericano de Ciencias Sociales un proyecto para la creación de la Comisión de Trabajo sobre Estudios Demográficos, expuso en la forma como a continuación se transcriben algunos de los problemas y necesidades de la investigación demográfica en América Latina al considerar los objetivos de esa comisión (la sintesis de esos objetivos se hizo en el párrafo 30):

En forma permanente tanto los organismos nacionales, como los internacionales, trabajan en el mejoramiento de las estadísticas continuas y periódicas; en tales propósitos es conveniente que se incluyan los puntos de vista de quienes utilizan la información al estudiar problemas demográficos, económicos y sociales. Igualmente diversas instituciones, principalmente nacionales, realizan estudios que por su importancia constituyen fuentes de información básica, aprovechables para análisis de otra naturaleza o con enfoques diferentes a los inicialmente propuestos. Es conveniente ampliar el uso de esta información.

En ocasiones se duplican esfuerzos considerables en materia de investigación, por no tomar en cuenta trabajos realizados con anterioridad. En otros casos los análisis no toman en consideración los resultados de otros trabajos, limitándose así la posibilidad de mayor profundidad y alcance de las propias conclusiones.

Los avances metodológicos se logran cada yez con mayor celeridad, entre otras causas, por el progreso general de las disciplinas sociales, disponiéndose así de instrumentos y metodologías con mayor poder explicativo de los fenómenos en estudio.

El contacto sistemático con los centros de investigación más avanzados dentro o fuera de la región debe ser la norma en las actividades, aunque es claro que el uso de cualquier metodología, también sistemáticamente, debe estar sujeto a la necesaria crítica y adecuación a las circumstancias particulares de la región.

Los requerimientos de profesionistas, particularmente los de alto nivel, no pueden ni deben ser satisfechos sólo por las universidades de los países desarrollados 0 por los centros regionales dependientes de organismos internacionales, Lo deseable es que estos últimos, a medida que se desarrollan centros de enseñanza e investigación de carácter nacional, se conviertan en organismos asesores de alta especialización.

Por otra parte, debe considerarse la conveniencia de que en algunos casos se establezcan centros que cubran las necesidades de varios países. Igualmente, debe cuidarse que los esfuerzos en la formación de profesionistas capaces, impliquen la permanente superación de los niveles académicos actuales, sin dejar de tomar como punto de referencia los progresos científicos en otras regiones.

Cada vez se hacen más necesarios el intercambio y la colaboración de los diversos centros latinoamericanos en la realización de proyectos comparables, a través de los cuales se amplían las posibilidades de explicación de los fenómenos sociales. Esto de ninguna manera elimina la realización necesaria de proyectos particulares en cada país, pero en ellos ha de tomarse en consideración la experiencia de los expertos de otros países latinoamericanos con el fin de mejorar la calidad de los trabajos.

En vista de las frecuentes limitaciones presupuestarias que afectan a la investigación, es necesario aprovechar al máximo los recursos disponibles. Asimismo debe aspirarse a que la investigación se realice con fondos

12 Carmen A. Miró, op. cit. 
cada vez más estables y de la propía región. La colaboración entre los diversos países latinoamericanos fortalecerá esta posición.

Los resultados de las investigaciones deben convertirse cada vez en mayor grado en instrumentos utílizables en la planeación del desarrollo. De aquí se desprende que las disciplinas sociales deben plantear sus conclusiones y las soluciones alternativas a los problemas, tomando en cuenta las necesidades del programador del desarrollo.

Lo anterior no significa que la investigación deba ser orientada exclusivamente por estas necesidades; esto limitaría considerablemente la libertad en la investigación científica y el desarrollo mismo de la ciencia; sino que dichas necesidades deben ser tomadas muy en cuenta, de tal forma que los estudios sean directamente útiles para el desarrollo económico y social, tanto a nivel nacional como regional interno.

En demografía, como en las demás ramas de las ciencias sociales, se da en cierta medida una dependencia respecto del mayor desarrollo científico en los países industrializados. Igual ocurre con los temas hacia los que se orienta la investigación misma. La toma de decisiones sobre los problemas a investigar y la independencia progresiva de recursos humanos y materiales, permitírá con mayor rapidez la elaboración de marcos teóricos aplicables a la realidad latinoamericana.

Por otra parte, debe tomarse en cuenta que la investigación, cuando se efectúa independientemente de la enseñanza, limita sus posibilidades de desarrollo y consecuentemente de lograr explicaciones más generales, en un marco de relatividad adecuado, de la problemática de la región y de los países en particular.

Asimismo, las actividades de investigación y enseñanza requieren de una institucionalización dinámica de las condiciones en que se realizan, que aseguren por una parte su permanencia y por otra su desarrollo, en donde las condiciones políticas particulares no interfieran la acción y en donde, a su vez, dichas condiciones políticas particulares sean tomadas en cuenta adecuadamente. 